\title{
BIFURCATION AND STABILITY OF PERIODIC SOLUTIONS FROM A ZERO EIGENVALUE
}

\author{
K. A. LANDMAN
}

(Received 28 April 1978)

\begin{abstract}
A study is made of the branching of time periodic solutions of a system of differential equations in $\mathbf{R}^{2}$ in the case of a double zero eigenvalue. It is shown that the solution need not be unique and the period of the solution is large. The stability of these solutions is analysed. Examples are given and generalizations to larger systems are discussed.
\end{abstract}

\section{Introduction}

Consider the two-dimensional system of differential equations

$$
\left.\begin{array}{l}
\frac{d x}{d t}=a(\mu) x+b(\mu) y+f^{(k)}(x, y)+f^{(k+1)}(x, y)+\ldots, \\
\frac{d y}{d t}=c(\mu) x+d(\mu) y+g^{(k)}(x, y)+g^{(k+1)}(x, y)+\ldots,
\end{array}\right\}
$$

where $\mu$ is a real parameter, $a, b, c, d$ are real analytic functions of $\mu$ for all $\mu \in \mathbf{R}$ and where the functions $f^{(j)}(x, y), g^{(j)}(x, y), j=k, k+1, \ldots(k \geqslant 2)$ are homogeneous polynomials in $x$ and $y$ of degree $j$. The variable $t$ is understood to represent time. Under these conditions $(x, y)=(0,0)$ is a solution pair for all values of $\mu$. In the usual way, the stability of the null solution is determined by the nature of the two eigenvalues $\lambda_{1}(\mu)$ and $\lambda_{2}(\mu)$ of

$$
\left[\begin{array}{ll}
a(\mu) & b(\mu) \\
c(\mu) & d(\mu)
\end{array}\right] .
$$

Suppose that for $\mu$ negative both $\lambda_{1}$ and $\lambda_{2}$ have negative real parts; then the null solution is asymptotically stable to small disturbances. Assume also that the null solution loses its stability at $\mu=0$; this means that at least one of the eigenvalues $\lambda_{1}$ or $\lambda_{2}$ crosses to the right half of the complex plane when $\mu$ passes through zero and becomes positive.

(C) Copyright Australian Mathematical Society 1979

Copyright. Apart from any fair dealing for scholarly purposes as permitted under the Copyright Act, no part of this JOURNAL may be reproduced by any process without written permission from the Treasurer of the Australian Mathematical Society. 
The classical bifurcation theorem due to Hopf [5], which generalizes results of Poincaré, assumes that the eigenvalues $\lambda_{1}$ and $\lambda_{2}$ are complex conjugates and that at $\mu=0$

$$
\lambda_{1}(0)=i \omega \neq 0 \text { and }\left.\frac{d}{d \mu} \operatorname{Re}\left(\lambda_{1}(\mu)\right)\right|_{\mu=0}>0 .
$$

The Hopf theorem tells us that there exists a solution of (1.1) which is periodic, with period close to $2 \pi / \omega$, when $|\mu|$ is small, and that this solution exists either for $\mu>0$ (supercritical) or for $\mu<0$ (subcritical) but not for both. Further this solution is unique, in particular there are no other periodic or nontrivial steady solutions in the neighbourhood of $(x, y)=(0,0)$ and $\mu=0$. Also, this periodic solution is stable if it is supercritical and unstable otherwise. This result of Hopf is valid for a system of $n$ ordinary differential equations, but it has an especially simple interpretation in two dimensions, when phase plane methods are available. A subcritical bifurcating periodic solution represents an unstable limit cycle around a stable focus, while a supercritical bifurcating periodic solution represents a stable limit cycle around un unstable focus.

In this article we will discuss the case when the null solution loses its stability when the first condition of (1.3) is not satisfied. Moreover, we do not require that $\lambda_{1}$ and $\lambda_{2}$ be complex for $\mu \neq 0$; they may be complex, real and possibly multiple. The assumptions made may be expressed as follows:

and either

$$
\operatorname{Re}\left(\lambda_{i}(\mu)\right)<0 \text { for } \mu<0, i=1,2,
$$

$$
\left.\begin{array}{rl}
\lambda_{1}(0)=0 \text { and }\left.\frac{d}{d \mu}\left(\operatorname{Re} \lambda_{1}(\mu)\right)\right|_{\mu=0}>0, \\
\text { or, for } i=1,2, \\
\lambda_{1}(0)=0 \text { and }\left.\frac{d}{d \mu}\left(\operatorname{Re} \lambda_{i}(\mu)\right)\right|_{\mu=0}>0 .
\end{array}\right\}
$$

There are two results which are known concerning this problem. A result by Coppel [2] for a quadratic system may be interpreted in the following way for the bifurcation problem. If $k=2$, then system (1.1) can only have a nonconstant periodic solution surrounding the origin and bifurcating from $\mu=0$, if the origin is a focus or a centre. Hence there can be no such periodic solution if the origin is a proper or improper node. Freedman [3], on the other hand, assuming (1.4), proves that no nonconstant periodic bifurcating solutions can exist with period $T(\mu) \rightarrow T_{0} \neq 0$ as $\mu \rightarrow 0$.

In this paper we will show that nonconstant periodic bifurcating solutions under (1.4) may exist if Freedman's requirement on the period is removed.

Under the conditions of loss of stability of the null solution at $\mu=0$ given in (1.4), the matrix (1.2) at $\mu=0$ can have only three possible structures, since either 
one or both of the eigenvalues $\lambda_{1}$ and $\lambda_{2}$ can be zero at $\mu=0$. Written in Jordan form these are easily shown to be

$$
\text { (A): }\left[\begin{array}{rr}
0 & 0 \\
0 & -1
\end{array}\right], \quad(B):\left[\begin{array}{ll}
0 & 0 \\
0 & 0
\end{array}\right], \text { or }(C):\left[\begin{array}{ll}
0 & 1 \\
0 & 0
\end{array}\right] \text {. }
$$

We wili show that periodic solutions may bifurcate in Cases (B) and (C), and that their periods will tend to infinity as $\mu \rightarrow 0$. Moreover there may be more than one such periodic solution and we may expect bifurcation of other steady solutions as well.

We present a simple example here which illustrates some of these properties. Consider the system

$$
\left.\begin{array}{l}
\frac{d x}{d t}=\mu \alpha x-(x+y)\left(x^{2}+y^{2}\right), \\
\frac{d y}{d t}=\mu \alpha y+(x-y)\left(x^{2}+y^{2}\right),
\end{array}\right\}
$$

where $\alpha$ is a positive real number. This is an example of Case (B) and the matrix, (1.2) has a double eigenvalue $\mu \alpha$, which is negative for $\mu<0$ and positive when $\mu>0$.

For convenience, let us rewrite (1.6) in polar coordinates $(r, \theta)$; put $x=r \cos \theta$, $y=r \sin \theta$, then the system (1.6) becomes

$$
\left.\begin{array}{l}
\frac{d r}{d t}=\mu \alpha r-r^{3} \\
\frac{d \theta}{d t}=r^{2} .
\end{array}\right\}
$$

The equation $d r / d t=0$ defines circles in the plane. This has the unique nontrivial solution $r^{2}=\mu \alpha$ which exists only for $\mu>0$. For this value of $r, d \theta / d t=\mu \alpha$; therefore without any loss in generality, since we are working with an autonomous system, we have $\theta=\mu \alpha t$. Hence a unique periodic solution of (1.7) is

$$
r=\sqrt{ }(\mu \alpha), \quad \theta=\mu \alpha t, \quad u>0,
$$

or, for (1.6),

$$
(x, y)=\sqrt{ }(\mu \alpha)(\cos (\mu \alpha t), \sin (\mu \alpha t)), \quad \mu>0 .
$$

This supercritical solution has period $2 \pi / \mu \alpha$ in $t$, which is large for all small values of $\mu$; in particular the period tends to infinity as $\mu \rightarrow 0$.

The steady solutions of (1.6) are found by setting the left side of this equation to zero. We see that this simple example only has $x=y=0$ as a steady solution.

The next three sections of this paper will be devoted respectively to a discussion of Cases (A), (B) and (C) defined in (1.5). In order to find small nonconstant 
periodic solutions $(x, y)$ which are continuously differentiable in time with period $T>0$, we introduce a small real parameter $\varepsilon>0$, whose value will be taken as a measure of the magnitude of the bifurcating $T$-periodic solution. Set

$$
(x, y)=\varepsilon(u, v)
$$

for small values of $\mu$; the explicit relation between $\mu$ and $\varepsilon$ will become apparent later. Substitute (1.10) into (1.1) and obtain

$$
\left.\begin{array}{l}
\frac{d u}{d t}=a(\mu) u+b(\mu) v+\varepsilon^{k-1} f^{(k)}(u, v)+\varepsilon^{k} f^{(k+1)}(u, v)+\ldots, \\
\frac{d v}{d t}=c(\mu) u+d(\mu) v+\varepsilon^{k-1} g^{(k)}(u, v)+\varepsilon^{k} g^{(k+1)}(u, v)+\ldots
\end{array}\right\}
$$

We suppose that at least one of $f^{(k)}(u, v), g^{(k)}(u, v)$ is not identically zero.

We will also study the stability of the bifurcating periodic orbits that we find. Suppose that $(\bar{u}(t), \bar{v}(t))$ is a nonconstant periodic solution of period $T$ of (1.11) whose orbit in the $(u, v)$ plane is $\Gamma$. We say that $\Gamma$ is asymptotically orbitally stable if any solution of (1.11) which comes near a point of $\Gamma$ tends to this orbit as $t \rightarrow \infty$ ([1], p. 323). The linear variational equations of this solution are

$$
\left.\begin{array}{l}
\frac{d u}{d t}=\left[a(\mu)+\varepsilon^{k-1} f_{u}^{(k)}(\bar{u}, \bar{v})\right] u+\left[b(\mu)+\varepsilon^{k-1} f_{v}^{(k)}(\bar{u}, \bar{v})\right] v+O\left(\varepsilon^{k}\right), \\
\frac{d v}{d t}=\left[c(\mu)+\varepsilon^{k-1} g_{u}^{(k)}(\bar{u}, \bar{v})\right] u+\left[d(\mu)+\varepsilon^{k-1} g_{v}^{(k)}(\bar{u}, \bar{v})\right] v+O\left(\varepsilon^{k}\right) .
\end{array}\right\}
$$

This is a linear system with coefficients periodic in $t$, period $T$. By Floquet theory ([1], p. $323 ;[4]$, p. 220$)$ the stability of $(\bar{u}, \bar{v})$ is determined by

$$
\exp \int_{0}^{T}\left[a(\mu)+d(\mu)+\varepsilon^{k-1}\left[f_{u}^{(k)}(\bar{u}, \bar{v})+g_{v}^{(k)}(\bar{u}, \bar{v})\right]\right] d s
$$

since one of the Floquet multipliers of this system is equal to one, corresponding to $(u, v)=(d \bar{u} / d t, d \bar{v} / d t)$. If the value of the expression (1.13) is less than one, $(\bar{u}, \bar{v})$ is asymptotically orbitally stable; but if it is greater than one, then the periodic orbit is orbitally unstable.

In Section 5 we give an account of generalizations to $n$-dimensional systems of equations and to equations in a Hilbert space.

\section{Case (A): a simple eigenvalue at $\mu=0$}

When the matrix (1.2) is evaluated at $\mu=0$ and has the form given in (1.5A), we may rewrite the system $(1.11)$ as

$$
\frac{d u}{d t}=\mu \bar{a}(\mu) u+\mu \bar{b}(\mu) v+\varepsilon^{k-1} f^{(k)}(u, v)+\varepsilon^{k} f^{(k+1)}(u, v)+\ldots
$$




$$
\frac{d v}{d t}=\mu \bar{c}(\mu) u+(-1+\mu \bar{d}(\mu)) v+\varepsilon^{k-1} g^{(k)}(u, v)+\varepsilon^{k} g^{(k+1)}(u, v)+\ldots
$$

where $\bar{a}, \bar{b}, \bar{c}$ and $\bar{d}$ are real analytic functions of $\mu$.

The following theorem allows us to eliminate $v$ of $(2.2)$ and to concentrate on equation (2.1).

THEOREM 2.1. Let $\varepsilon$ and $\mu$ be sufficiently small and let $u(t)$ belong to the space of continuously differentiable functions of $t$, which are T-periodic for some $T>0$ such that $\max _{0 \leqslant t \leqslant T}|u(t)| \leqslant 1$. Then equation (2.2) has a unique solution $\vec{v}=\bar{v}(t, u, \mu, \varepsilon)$ which is $T$-periodic, with $\max _{0 \leqslant t \leqslant T}|\bar{v}(t)| \leqslant \beta$ where $\beta=O\left(\mu, \varepsilon^{k-1}\right)$ as $\mu, \varepsilon \rightarrow 0$. Moreover

$$
\bar{v}(t, u, \mu, \varepsilon)=\hat{v}(t, u, \mu, \varepsilon)+o\left(\mu, \varepsilon^{k-1}\right) \quad \text { as } \mu, \varepsilon \rightarrow 0,
$$

where $\hat{v}$ is the T-periodic solution of

$$
\frac{d v}{d t}=-v+\mu \bar{c}(0) u+\varepsilon^{k-1} g^{(k)}(u, 0)
$$

Also, $\bar{v}$ is an analytic function of $u, \mu$ and $\varepsilon$.

(We note that since $\varepsilon$ was chosen as a measure of the norm of the solution we lose no generality by choosing the magnitude of $u$ to be less than or equal to one.)

PROOF. Write (2.2) as

$$
\frac{d v}{d t}=-v+p(t)+q(t, v, \mu, \varepsilon)
$$

where

$$
\begin{gathered}
p(t)=\mu \bar{c}(0) u(t)+\varepsilon^{k-1} g^{(k)}(u(t), 0), \\
q(t, v, \mu, \varepsilon)=\mu d(\mu) v+\mu(\bar{c}(\mu)-\bar{c}(0)) u(t) \\
+\varepsilon^{k-1}\left\{g^{(k)}(u, v)-g^{(k)}(u, 0)\right\}+\varepsilon^{k} g^{(k+1)}(u, v)+\ldots .
\end{gathered}
$$

For each $T$-periodic function $u(t), p(t)$ is a known function of $t$ which is also $T$-periodic.

First let us consider the nonhomogeneous linear equation

$$
\frac{d v}{d t}=-v+p(t)
$$

Since the linear equation $d v / d t=-v$ has no $T$-periodic solution other than $v=0$, it is called noncritical with respect to the $T$-periodic functions. Then it is well known ([4], p. 148) that (2.8) has a unique $T$-periodic solution $\hat{v}$ such that

$$
\max _{0 \leqslant t \leqslant T}|\hat{v}| \leqslant \text { constant }\left\{\max _{0 \leqslant t \leqslant T}|p(t)|\right\}=O\left(\mu, \varepsilon^{k-1}\right) .
$$


Now return to $(2.5)$ and write

then $z$ satisfies

$$
v=\hat{b}+z
$$

$$
\frac{d z}{d t}=-z+q(t, \hat{v}+z, \mu, \varepsilon)
$$

Since $q$ satisfies the required Lipschitz conditions, we know that there exists a unique $T$-periodic solution

$$
\bar{v}=\hat{v}(t, u, \mu, \varepsilon)+z(t, u, \mu, \varepsilon),
$$

where $|z(t, u, \mu, \varepsilon)|=o\left(\mu, \varepsilon^{k-1}\right)$. This follows from [4] (pp. 154-156). Finally since $p$ and $q$ are analytic functions of $u, \mu$ and $\varepsilon$, so is $\bar{v}$. This completes the proof.

This theorem allows $v$ in equation (2.1) to be replaced by $\bar{v}$, the solution of (2.2). Then we see that the problem of solving the pair of equations (2.1-2.2) for nonconstant $T$-periodic solutions reduces to solving the following equation for nonconstant $T$-periodic solutions $u(t)$ with $\max _{0 \leqslant t \leqslant T}|u(t)| \leqslant 1$, provided $\varepsilon$ and $\mu$ are sufficiently small to satisfy Theorem 1.1 :

$$
\frac{d u}{d t}=\mu \bar{a}(\mu) u+\mu \bar{b}(\mu) \bar{v}(t, u, \mu, \varepsilon)+\varepsilon^{k-1} f^{(k)}(u, \bar{v})+\varepsilon^{k} f^{(k+1)}(u, \bar{v})+\ldots
$$

We are looking for a solution $u(t)$ which can be expanded as a series in $\varepsilon$ :

$$
u=u_{0}+\varepsilon u_{1}+\varepsilon^{2} u_{2}+\ldots,
$$

where the $u_{i}(i=0,1,2, \ldots)$ are nonconstant $T$-periodic and bounded as $\varepsilon \rightarrow 0$. Substitution of (2.13) into (2.12) tells us that the nonconstant $T$-periodic $u_{0}$ must satisfy

$$
\frac{d u_{0}}{d t}=\mu a(0) u_{0}+\varepsilon^{k-1} f^{(k)}\left(u_{0}, 0\right),
$$

where the terms on the right are the dominant linear and nonlinear terms. But $f^{(k)}\left(u_{0}, 0\right)=A u_{0}^{k}$, so that we must find solutions of

$$
\frac{d u_{0}}{d t}=\mu a(0) u_{0}+\varepsilon^{k-1} A u_{0}^{k}
$$

It is obvious that this equation has no nonconstant $T$-periodic solution. Hence it follows that no such solution of the form (2.13) can exist. This implies that when the linear part of (1.1) evaluated at $\mu=0$ has the form represented by Case (A), no periodic solution (of any period) can exist.

3. Case (B): a double eigenvalue at $\mu=0$

In this case, the coefficients of the linear terms in equation (1.11) as given by 
matrix (1.2) are

$$
\left[\begin{array}{ll}
\mu \bar{a}(\mu) & \mu \bar{b}(\mu) \\
\mu \bar{c}(\mu) & \mu \bar{d}(\mu)
\end{array}\right],
$$

where $\bar{a}, \bar{b}, \bar{c}$ and $\bar{d}$ are analytic functions of $\mu$. For $|\mu|$ small the two eigenvalues of (3.1) are analytic functions of $\mu$ and may be written as

$$
\left.\begin{array}{l}
\lambda_{1}=\mu \kappa_{1}(\mu)=\mu\left(\kappa_{11}+\mu \kappa_{12}+\mu^{2} \kappa_{13}+\ldots\right), \\
\lambda_{2}=\mu \kappa_{2}(\mu)=\mu\left(\kappa_{21}+\mu \kappa_{22}+\mu^{2} \kappa_{23}+\ldots\right),
\end{array}\right\}
$$

where the $\kappa_{i j}$ are complex coefficients. In order to satisfy condition (1.4) the first nonvanishing power of $\mu$ must be odd; for convenience, so as to minimize the technicalities, we assume that $\kappa_{11}, \kappa_{21} \neq 0$. (The more general case can be investigated in a similar fashion.) That is, we assume that the dominant terms of (3.1) are given by

$$
\left[\begin{array}{ll}
\mu a & \mu b \\
\mu c & \mu d
\end{array}\right],
$$

where $a=\bar{a}(0), b=\bar{b}(0), c=\bar{c}(0)$ and $d=\bar{d}(0)$. The assumption on the stability of the null solution given by condition (1.4) is equivalent to

$$
a+d>0, a d-b c>0 .
$$

Therefore the linearized system of (1.11) cannot have any periodic or constant solutions for $|\mu|$ small. We are looking for a nonconstant $T$-periodic solution $(u, v)$ of (1.11) which is a function of $\mu$; such a solution can only be generated by a balance between the linear and nonlinear terms. Since the dominant terms in (1.11) are

$$
\left.\begin{array}{l}
\frac{d u}{d t}=\mu a u+\mu b v+\varepsilon^{k-1} f^{(k)}(u, v) \\
\frac{d v}{d t}=\mu c u+\mu d v+\varepsilon^{k-1} g^{(k)}(u, v)
\end{array}\right\}
$$

this suggests that we set

$$
\mu=\varepsilon^{k-1} v, \quad|v|=0(1) \text { as } \varepsilon \rightarrow 0 .
$$

Then (3.5) becomes

$$
\left.\begin{array}{l}
\frac{d u}{d t}=\varepsilon^{k-1}\left[v a u+v b v+f^{(k)}(u, v)\right] \\
\frac{d v}{d t}=\varepsilon^{k-1}\left[v c u+v d v+g^{(k)}(u, v)\right]
\end{array}\right\}
$$


In order to find the $T$-periodic solution $(u, v)$ we need to rescale the time variable $t$; we obtain an equation which is $O(1)$ in $\varepsilon$ if we put

$$
s=\varepsilon^{k-1} t
$$

then we have

$$
\left.\begin{array}{l}
\frac{d u}{d s}=v a u+v b v+f^{(k)}(u, v) \\
\frac{d v}{d s}=v c u+v d v+g^{(k)}(u, v)
\end{array}\right\}
$$

We are now looking for solutions $(u(s), v(s))$ which are periodic in $s$, period $\widetilde{T}=O(1)$ as $\varepsilon \rightarrow 0$. The relation between the period $T$ in $t$ and $\tilde{T}$ in $s$ can be seen from (3.8): $T=\tau / \varepsilon^{k-1}$; hence the period in the original time $t$ will be large for small values of $\varepsilon$.

Now let us suppose that the reduced nonlinear equation (3.9) has a periodic solution $(\bar{u}(s, v), \bar{v}(s, v))$, period $\tilde{T}(v)$ in $s$. When does the full equation (1.11), satisfying (1.4) (or equivalently (3.4)) have a periodic solution close to $(\bar{u}, \bar{v})$ and how is its period related to $\widetilde{T}$ ? To answer these questions we need to study the variational equations of this solution:

$$
\left.\begin{array}{l}
\frac{d \phi}{d s}=\left(v a+f_{u}^{(k)}(\bar{u}, \bar{v})\right) \phi+\left(v b+f_{v}^{(k)}(\bar{u}, \bar{v})\right) \psi, \\
\frac{d \psi}{d s}=\left(v c+g_{u}^{(k)}(\bar{u}, \bar{v})\right) \phi+\left(v d+g_{v}^{(k)}(\bar{u}, \bar{v})\right) \psi
\end{array}\right\}
$$

In the usual way ([4], p. 120) one solution of $(3.10)$ is just $(\phi, \psi)=(d \bar{u} / d s, d \bar{v} / d s)$, and since this is a $\tilde{T}$-periodic solution, it corresponds to a Floquet multiplier having the value one. The second multiplier can then be determined by the trace of the variational matrix ([4], p. 120) and is

$$
\exp \int_{0}^{T}\left[v(a+d)+f_{u}^{(k)}(\bar{u}(s), \bar{v}(s))+g_{v}^{(k)}(\bar{u}(s), \bar{v}(s))\right] d s .
$$

THEOREM 3.1. Suppose that the reduced system (3.9) has a nonconstant T-periodic solution $(\tilde{u}(s), v), \bar{v}(s), v))$ in $s=\varepsilon^{k-1} t$. Then if the value of the expression (3.11) is not equal to one, the full perturbed system (1.1), satisfying (1.5B) has a unique small bifurcating nonconstant periodic solution in the neighbourhood of $\mu=0$. Its period is $T^{*}(\varepsilon) / \varepsilon^{k-1}$ in $t$, where $T^{*}(0)=T$ and its structure is

where

$$
\left.\begin{array}{l}
\left(x^{*}(t, v, \varepsilon), y^{*}(t, v, \varepsilon)\right)=\varepsilon\left(u^{*}(t, v, \varepsilon), v^{*}(t, v, \varepsilon)\right), \quad \mu=\varepsilon^{k-1} v, \\
\left(u^{*}(t, v, 0), v^{*}(t, v, 0)\right)=\left(\bar{u}\left(\varepsilon^{k-1} t, v\right), \bar{v}\left(\varepsilon^{k-1} t, v\right)\right)=(\bar{u}(s, v), \bar{v}(s, v)) .
\end{array}\right\}
$$


If the value of the expression (3.11) is less than one, then for $\varepsilon$ sufficiently small, $\left(x^{*}, y^{*}\right)$ is asymptotically orbitally stable. If the value of the expression (3.11) is greater than one, $\left(x^{*}, y^{*}\right)$ is orbitally unstable.

Proof. Using $(x, y)=\varepsilon(u, v)$ of $(1.10)$, we can, with the aid of (3.3), write the full perturbed system $(1,11)$ as

$$
\left.\begin{array}{l}
\frac{d u}{d t}=\varepsilon^{k-1}\left(v a u+v b v+f^{(k)}(u, v)+\varepsilon R_{1}(u, v, \varepsilon)\right), \\
\frac{d v}{d t}=\varepsilon^{k-1}\left(v c u+v d v+g^{(k)}(u, v)+\varepsilon R_{2}(u, v, \varepsilon)\right),
\end{array}\right\}
$$

where

$$
\left.\begin{array}{l}
R_{1}(u, v, \varepsilon)=\varepsilon^{k-2} v\{[\bar{a}(\mu)-a] u+[\bar{b}(\mu)-b] v\}+f^{(k+1)}(u, v)+\varepsilon f^{(k+2)}(u, v)+\ldots, \\
R_{2}(u, v, \varepsilon)=\varepsilon^{k-2} v\{[\bar{c}(\mu)-c] u+[\bar{d}(\mu)-d] v\}+g^{(k+1)}(u, v)+\varepsilon g^{(k+2)}(u, v)+\ldots .
\end{array}\right\}
$$

Now, $R_{1}$ and $R_{2}$ are continuous in $u, v$ and $\varepsilon$ and they have continuous partial derivatives with respect to $u$ and $v$, and $R_{1}(u, v, 0)$ and $R_{2}(u, v, 0)$ are bounded. The existence and uniqueness of such a solution $\left(u^{*}, v^{*}\right)$ to $(3.13)$ follows from the theory of perturbations of autonomous systems ([1], p. 352; [4], p. 222; [9], p. $95)$. Further, the stability of $(\bar{u}, \bar{v})$ is determined by (3.11), which are the dominant terms in the multiplier (1.13) whose value determines the stability of $\left(x^{*}, y^{*}\right)$.

The following theorem may also be proved; it is useful in proving the nonexistence of solutions to (1.1).

THEOREM 3.2. If $\left(x^{*}(t, \mu, \varepsilon), y^{*}(t, \mu, \varepsilon)\right)$ is a nonconstant small $T(\varepsilon)$-periodic solution bifurcating from $\mu=0$ of (1.1), then a nonconstant $\widetilde{T}$-periodic solution in $s=\varepsilon^{k-1} t$ of the reduced problem (3.9) must exist, where $\tilde{T}=\varepsilon^{k-1} T(0)$.

Proof. We can write this solution $\left(x^{*}, y^{*}\right)$ as

$$
\left(x^{*}, y^{*}\right)=\varepsilon\left(u^{*}(t, \mu, \varepsilon), v^{*}(t, \mu, \varepsilon)\right)
$$

and since (3.15) is a nonconstant $T(\varepsilon)$-periodic solution, we can expand $u^{*}$ and $v^{*}$ in powers of $\varepsilon$

$$
\left.\begin{array}{l}
u^{*}(t, \mu, \varepsilon)=u_{0}(t, \mu, \varepsilon)+\varepsilon u_{1}(t, \mu, \varepsilon)+\ldots, \\
v^{*}(t, \mu, \varepsilon)=v_{0}(t, \mu, \varepsilon)+\varepsilon v_{1}(t, \mu, \varepsilon)+\ldots,
\end{array}\right\}
$$

where the coefficients $u_{i}, v_{l}(i=0,1,2, \ldots)$ are nonconstant $T(\varepsilon)$-periodic and in particular we require $u_{0}$ and $v_{0}$ to be dependent on $\mu$. The discussion prior to Theorem 3.1 requires $\left(u_{0}, v_{0}\right)$ to be a solution to (3.9). 
Therefore we can now focus our attention on the system (3.9). First of all, from the discussion of the stability of the null solution, the matrix

$$
\left[\begin{array}{ll}
a & b \\
c & d
\end{array}\right]
$$

satisfies (3.4). This matrix can be written in Jordan form, and it is easy to establish that there are only four possible structures of (3.17). These are

$$
\left[\begin{array}{ll}
\alpha & 0 \\
0 & \alpha
\end{array}\right],\left[\begin{array}{ll}
\alpha & 0 \\
0 & \beta
\end{array}\right],\left[\begin{array}{ll}
\alpha & 1 \\
0 & \alpha
\end{array}\right] \text { or }\left[\begin{array}{cc}
\alpha & \gamma \\
-\gamma & \alpha
\end{array}\right], \alpha, \beta>0 .
$$

Therefore the origin $(u, v)=(0,0)$ of $(3.9)$ is either a node (proper or improper node) or a focus.

For quadratic systems in the phase plane, that is for $k=2$, it is known [2] that a critical point in the interior of a closed orbit must be either a focus or a centre. Therefore, if we have a node at the origin no closed orbit can surround it. However, this result is not true for cubic systems-this is illustrated by the example given in the Introduction.

Example 1. Suppose the system (3.9) has the structure

$$
\left.\begin{array}{l}
\frac{d u}{d s}=v \alpha u-(u+v)\left(u^{2}+v^{2}\right), \\
\frac{d v}{d s}=v \alpha v+(u-v)\left(u^{2}+v^{2}\right), \quad s=\varepsilon^{2} t, \quad \mu=\varepsilon^{2} v, \quad \alpha>0 .
\end{array}\right\}
$$

We found in the Introduction that this system has a unique supercritical closed orbit surrounding the proper node at the origin, $\bar{u}^{2}+\bar{v}^{2}=v \alpha(v>0)$, with period $2 \pi / v \alpha$ in $s$.

The variational system for this periodic solution is

$$
\left[\begin{array}{l}
\frac{d \phi}{d s} \\
\frac{d \psi}{d s}
\end{array}\right]=\left[\begin{array}{cc}
\left(v \alpha-3 \bar{u}^{2}-\bar{v}^{2}-2 \bar{u} \bar{v}\right) & \left(-3 \bar{v}^{2}-\bar{u}^{2}-2 \bar{u} \bar{v}\right) \\
\left(3 \bar{u}^{2}+\bar{v}^{2}-2 \bar{u} \bar{v}\right) & \left(v \alpha-3 \bar{v}^{2}-\bar{u}^{2}+2 \bar{u} \bar{v}\right)
\end{array}\right]\left[\begin{array}{l}
\phi \\
\psi
\end{array}\right] .
$$

The second Floquet multiplier, as given by (3.11), is just

$$
\exp \int_{0}^{2 \pi / v \alpha}\left[2 v \alpha-4\left(\bar{u}^{2}(s)+\bar{v}^{2}(s)\right)\right] d s=e^{-4 \pi}
$$

which is less than one. Therefore the full equation (1.1) has a nonconstant periodic 
solution in the neighbourhood of

$$
\sqrt{ }(\mu \alpha)(\cos (\mu \alpha t), \sin (\mu \alpha t)), \quad \mu>0,
$$

with period close to $2 \pi / \mu \alpha$ and this solution is asymptotically orbitally stable.

This raises some interesting questions. Suppose system (3.9) has a node at the origin. We know that for a quadratic system there exists at least one nonzero critical point, but that no periodic solution can surround the origin. This means that there exists at least one steady bifurcating solution but no nonconstant periodic bifurcating solution. On the other hand, from the above example and others to follow, it can be seen that for systems which are not quadratic a nonconstant periodic bifurcating solution can exist. But in the examples given the null solution is the only steady solution. This allows us to pose the following question. For a double eigenvalue of type (B) at $\mu=0$, does there always exist a bifurcating solution from $(x, y)=(0,0), \mu=0$ which is either steady or nonconstant periodic? If there is a steady solution, does this exclude the existence of a nonconstant periodic one and vice-versa? We have not been able to construct any examples where neither steady nor periodic bifurcating solutions exist or where both exist together.

To throw some light on this we suppose that there exists a closed orbit surrounding the origin. Thus it encloses at least one critical point as required. The index of an isolated critical point is defined ([1], p. 400) and equals -1 if it is a saddle point and +1 otherwise. Further, the interior of any closed orbit contains a finite number of critical points, the sum of whose indices is one. Suppose we have a cubic system, then including the origin, there are at most four critical points. The above discussion tells us that a closed orbit surrounding the origin cannot enclose the origin and one or three other isolated critical points, for then the sum of the indices will either be zero or even. This partially answers the above questions, but does not exclude the possibility of three isolated critical points inside a closed orbit. Since the index of an isolated critical point exterior to the closed orbit is not defined, the above discussion tells us nothing about the existence or nonexistence of such points.

Further, the question regarding the number of bifurcating periodic solutions is still open [2]. This contrasts the uniqueness result by Hopf [5].

Finally, we give an example where the linear part is a focus.

Example 2. Consider the problem when (3.9) has the following structure:

$$
\left.\begin{array}{l}
\frac{d u}{d s}=v \alpha u+v \gamma v-u\left(u^{2}+v^{2}\right), \\
\frac{d v}{d s}=-v \gamma u+v \alpha v-v\left(u^{2}+v^{2}\right), \quad s=\varepsilon^{2} t, \quad \mu=\varepsilon^{2} v, \quad \alpha>0, \quad \gamma \neq 0 .
\end{array}\right\}
$$


The null solution loses its stability as the complex eigenvalues $\mu(\alpha \pm i \gamma)+O\left(\mu^{2}\right)$ of (1.5) pass from the left half of the complex plane to the right half.

In polar coordinates $x=r \cos \theta, y=r \sin \theta$, this system becomes

$$
\left.\begin{array}{l}
\frac{d r}{d s}=v \alpha r-r^{3} \\
\frac{d \theta}{d s}=-v \gamma .
\end{array}\right\}
$$

The equation $d r / d s=0$ defines a circle $r^{2}=v \alpha$ which exists only for $v>0$. Also, without loss of generality $\theta=-v \gamma s$; hence a unique supercritical periodic solution is

$$
r=\sqrt{ }(\nu \alpha), \quad \theta=-v \gamma s, \quad v>0,
$$

or

$$
\bar{u}=\sqrt{ }(v \alpha) \cos (v \gamma s), \quad \bar{v}=-\sqrt{ }(v \alpha) \sin (v \gamma s), \quad v>0 .
$$

The variational system for this periodic solution is

$$
\left[\begin{array}{l}
\frac{d \phi}{d s} \\
\frac{d \psi}{d s}
\end{array}\right]=\left[\begin{array}{cc}
v \alpha-3 \bar{u}^{2}-\bar{v}^{2} & v \gamma-2 \bar{u} \bar{v} \\
-v \gamma-2 \bar{u} \bar{v} & v \alpha-3 \bar{v}^{2}-\bar{u}^{2}
\end{array}\right]\left[\begin{array}{l}
\phi \\
\psi
\end{array}\right] .
$$

Since the trace of variational matrix in (3.27) is the same as that in Example 1, there is a unique periodic solution in the neighbourhood of

$$
\sqrt{ }(\mu \alpha)(\cos (\mu \gamma t),-\sin (\mu \gamma t)), \quad \mu>0,
$$

and its period is close to $2 \pi / \mu \gamma$. This solution is asymptotically orbitally stable.

Further, no steady solutions exist.

\section{Case (C): second type of double eigenvalue}

In the final case, the coefficients of the linear terms in equation (1.11) as given by matrix (1.2) are

$$
\left[\begin{array}{cc}
\mu \bar{a}(\mu) & 1+\mu \bar{b}(\mu) \\
\mu \bar{c}(\mu) & \mu \bar{a}(\mu)
\end{array}\right],
$$

where $\bar{a}, \bar{b}, \bar{c}$ and $d$ are analytic functions of $\mu$. For $|\mu|$ small, the two eigenvalues of (4.1) are functions of $|\mu|^{ \pm}$and may be written as

$$
\left.\begin{array}{l}
\lambda_{1}=|\mu|^{\frac{1}{2}} \tau_{11}+\mu \tau_{12}+|\mu|^{\frac{1}{2}} \tau_{13}+\ldots, \\
\lambda_{2}=|\mu|^{\frac{1}{2}} \tau_{21}+\mu \tau_{22}+|\mu|^{\frac{1}{2}} \tau_{23}+\ldots,
\end{array}\right\}
$$


where the $\tau_{i j}$ are complex coefficients. To minimize technicalities we write

$$
a=\bar{a}(0), \quad b=\bar{b}(0), \quad d=\bar{d}(0), \quad c_{0}=\bar{c}(0), \quad c_{1}=\left.\frac{d}{d \mu}(\bar{c}(\mu))\right|_{\mu=0},
$$

and assume that $a, b, d$ and either $c_{0}$ or $c_{1}$ are nonzero real numbers. Then consider the dominant terms of the matrix (4.1) which determine the stability behaviour of the null solution:

$$
\left[\begin{array}{ccc}
\mu a & 1 \\
\mu c_{0}+\mu^{2} c_{1} & \mu d
\end{array}\right] .
$$

The eigenvalues of matrix (4.4) are

$$
\lambda^{ \pm}=\frac{1}{2}\left\{\mu(a+d) \pm \sqrt{ }\left[4 \mu c_{0}+\mu^{2}\left((a+d)^{2}-4\left(a d-c_{1}\right)\right)\right]\right\} .
$$

For $\mu<0$, both $\lambda^{ \pm}$must have negative real parts and at least one of these must have positive real part when $\mu>0$. Since $\mu c_{0}$ is the dominant term under the square root, we need the following two alternative conditions:

$$
\begin{aligned}
& C 1 \text { if } c_{0}=0 \text { and } c_{1} \neq 0, a+d>0, a d-c_{1}>0, \\
& C 2 \text { if } c_{0} \neq 0, a+d>0, c_{0}>0 .
\end{aligned}
$$

Thus we see that if $C 2$ is satisfied, then for $\mu$ negative disturbances to the null solution are decaying oscillations, while for $\mu$ positive they are growing exponentials. In contrast to this, if $C l$ is satisfied, for $\mu$ negative disturbances may be decaying exponentials (if $(a-d)^{2} \geqslant 4 c_{1}$ ) and for $\mu$ positive they are growing exponentials. This difference in the nature of the behaviour of the disturbances affects whether (1.11) has periodic solutions or not.

First of all, we see from equations (1.11) that $v$ must tend to zero as $\mu$ and $\varepsilon$ tend to zero. This allows us to seek solutions of the form

$$
u=\hat{u}, \quad v=\varepsilon^{\sigma} \hat{v}, \quad \mu=\varepsilon^{\beta} v, \quad \sigma, \beta>0,
$$

then (1.11) becomes

$$
\left.\begin{array}{l}
\frac{d \hat{u}}{d t}=\varepsilon^{\beta} v a \hat{u}+\varepsilon^{\sigma} \hat{v}+\varepsilon^{k-1} f^{(k)}(u, 0)+o\left(\varepsilon^{\beta}, \varepsilon^{\sigma}, \varepsilon^{k-1}\right), \\
\frac{d \hat{v}}{d t}=\varepsilon^{\beta-\sigma} v c_{0} \hat{u}+\varepsilon^{2 \beta-\sigma} v c_{1} \hat{u}+\varepsilon^{\beta} v d \hat{v}+\varepsilon^{k-1-\sigma} g^{(k)}(u, 0)+o\left(\varepsilon^{\beta}, \varepsilon^{2 \beta-\sigma}, \varepsilon^{k-1-\sigma}\right) .
\end{array}\right\}
$$

We now distinguish between the two cases $C 1$ and $C 2$.

(1) When $C l$ is satisfied. We are looking for a nonconstant $T$-periodic solution which is a function of $\mu$. Since the linear terms of (4.7) do not give such a solution, 
a balance between the linear and nonlinear terms is needed. This suggests that we set

$$
\sigma=\beta=(k-1) / 2 .
$$

Then the dominant terms in these equations are

$$
\left.\begin{array}{l}
\frac{d \hat{u}}{d t}=\varepsilon^{(k-1) / 2}[v a \hat{u}+\hat{v}], \\
\frac{d \hat{v}}{d t}=\varepsilon^{(k-1) / 2}\left[v^{2} c_{1} \hat{u}+v d \hat{v}+B \hat{u}^{k}\right],
\end{array}\right\}
$$

for $(\hat{u}(t), \hat{v}(t)) T$-periodic for some $T>0$. We have replaced $g^{(k)}(\hat{u}, 0)$ by $B \hat{u}^{k}$ here. Now, Bendixon's theorem [8] gives a necessary condition for a periodic solution to exist in the plane; the trace of the variational matrix corresponding to (4.9) must have a zero. However, from (4.9), we see that the trace equals $\varepsilon^{k-1} v(a+d)$. Condition $C 1$ requires $a+d>0$, hence for $v \neq 0$ Bendixon's condition can never be satisfied. Therefore the system (4.9) cannot have any closed orbits if we require the loss of stability on the null solution. By the same reasoning as in Theorem 3.2, this tells us that (1.1) cannot have any bifurcating nonconstant periodic solutions. (It can be shown, [6] and [7], that a unique steady bifurcating solution exists which is unstable on both sides of criticality.)

(2) When C2 is satisfied. This time the system (4.7) suggests that we choose

$$
\sigma=(k-1) / 2 \text { and } \beta=(k-1),
$$

then the dominant terms are

$$
\left.\begin{array}{l}
\frac{d \hat{u}}{d t}=\varepsilon^{(k-1) / 2} v \\
\frac{d \hat{v}}{d t}=\varepsilon^{(k-1) / 2}\left(v c_{0} \hat{u}+B \hat{u}^{k}\right),
\end{array}\right\}
$$

for $(\hat{u}(t), \hat{v}(t)) T$-periodic in $t$. It is necessary to rescale the time variable in order to obtain a system which is $O(1)$ in $\varepsilon$. Put

$$
s=\varepsilon^{(k-1) / 2} t
$$

for then we obtain

$$
\left.\begin{array}{l}
\frac{d \hat{u}}{d s}=v, \\
\frac{d \hat{v}}{d s}=v c_{0} \hat{u}+B \hat{u}^{k},
\end{array}\right\}
$$

for $(\hat{u}(s), \hat{v}(s)) \hat{T}$-periodic in $s$, where $\hat{T}=O(1)$ as $\varepsilon \rightarrow 0$. Then $T=\tilde{T} / \varepsilon^{(k-1) / 2}$; hence 
the period of the solution in $t$ will be large. Such periodic solutions do exist-an example will be presented below.

Let $(\bar{u}, \bar{v})$ be such a $\tilde{T}$-periodic solution. The variational matrix corresponding to this solution is

$$
\left[\begin{array}{cc}
0 & 1 \\
v c_{0}+k B \bar{u}^{k-1} & 0
\end{array}\right],
$$

this has both elements on the leading diagonal equal to zero; that is, both the Floquet multipliers are equal to one. Therefore, the solutions form nonisolated curves in the $(\hat{u}, \hat{v})$ plane and the theorems we have used in Section 3 do not apply. The system (4.13) is called a fully oscillatory system. Urabe [9] analyses the problem of when a perturbation of a fully oscillatory system has a unique closed orbit. Using the local coordinates of a moving orthonormal system, Urabe writes the perturbed system as an averaged equation. If this has a simple zero then a unique orbit exists.

Let us consider the example when $k=3$. First of all, with the condition $C 2$ and $B$ negative, the system (4.13) has two critical points for $v>0$, namely

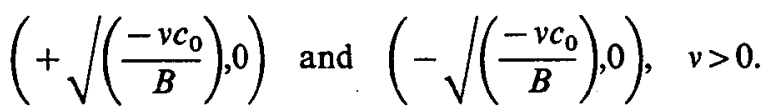

These are the points $\mathrm{A}$ and $\mathrm{C}$ marked in Fig. 1. Therefore it can be shown, [6, 7], that the system (1.1) has a branch of steady bifurcating supercritical solutions,

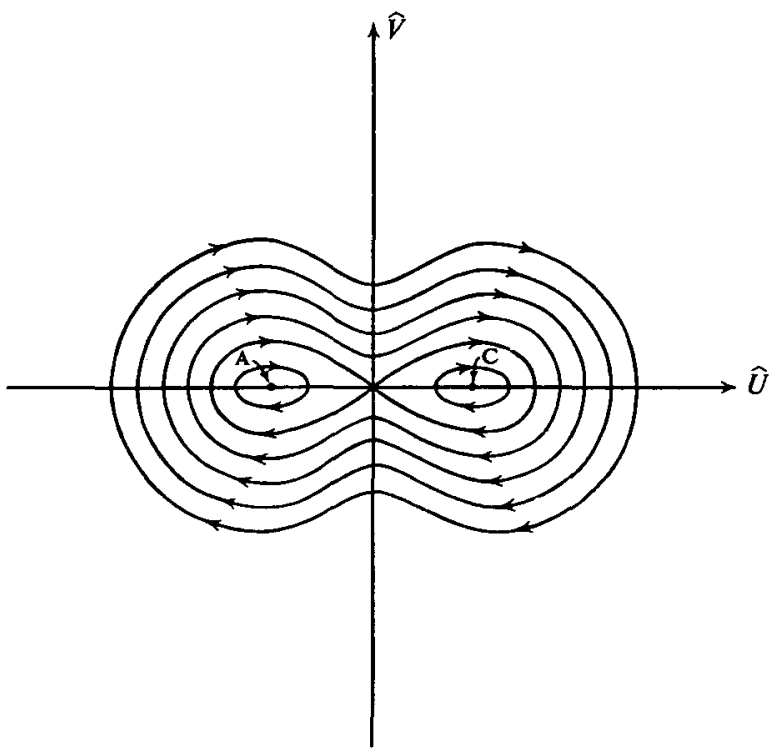

Fig. 1. Illustrating the phases for different values of $\boldsymbol{H}$. 
which is close to

$$
\left( \pm \sqrt{ }\left(\frac{-\mu c_{0}}{B}\right), 0\right), \quad \mu>0
$$

Also, the system (4.13) is a conservative system and has first integral

$$
\frac{1}{2} v c_{0} \hat{u}^{2}+\frac{1}{4} B \hat{u}^{4}-\frac{1}{2} \hat{v}^{2}=-H,
$$

where $H>0$ are constants. The phase portrait for different values of $H$ is as shown in Fig. 1. Thus we have nonisolated closed curves in the $(\hat{u}, \hat{v})$ plane-both the Floquet multipliers of the variational system are equal to one.

We remark that this example shows that in Case (C) both steady and periodic bifurcating solutions may exist together. This should be compared with the discussion of Case (B) in Section 3.

\section{Generalizations}

Under conditions which are generalizations of the ones used in the Introduction, the results which we have obtained can be extended to the bifurcation of nonconstant periodic solutions of a system of $n$ ordinary differential equations.

Consider the system

$$
\frac{d x}{d t}=A(\mu) x+F(x)
$$

where $x$ and $F$ are $n$ vectors and $A$ is an $n \times n$ matrix. Assume that $A$ is analytic in $\mu$ for all $\mu \in \mathbf{R}$ and that $F$ is an analytic nonlinear function of $x$ for small $|x|$.

The matrix $A$ can always be written in Jordan canonical form

$$
\left[\begin{array}{cc}
\bar{A}(\mu) & 0 \\
0 & B(\mu)
\end{array}\right],
$$

where $\bar{A}(\mu)$ is an $r \times r$ matrix and $B(\mu)$ is an $(n-r) \times(n-r)$ matrix. We make the following assumptions. For $\mu$ negative all the diagonal elements (which are the eigenvalues of $A(\mu)$ ) have negative real parts; at $\mu=0$, the diagonal elements of $\bar{A}(0)$ are zero while those of $B(0)$ have negative real parts; and for small positive $\mu$, at least one of the diagonal elements of $\bar{A}(\mu)$ is positive while those of $B(\mu)$ still remain in the left half of the complex plane. Hence the null solution loses its stability through the zero eigenvalue which has multiplicity $r$.

A standard procedure, which is just a generalization of Theorem 2.1 using perturbation techniques and the contraction mapping theorem ([4], pp. 148-156), gives that the existence of nonconstant $T$-periodic solutions depends only on the 
system of $r$ ordinary differential equations

$$
\frac{d y}{d t}=\bar{A}(\mu) y+\bar{F}(y)
$$

where $y=\left(x_{1}, x_{2}, \ldots, x_{r}\right)$, the first $r$ components of $x$ and

$$
\bar{F}(y)=\left(F_{1}\left(x_{1}, \ldots, x_{r}, 0, \ldots, 0\right), F_{2}\left(x_{1}, \ldots, x_{r}, 0, \ldots, 0\right), \ldots, F_{r}\left(x_{1}, \ldots, x_{r}, 0, \ldots, 0\right)\right)
$$

where $F_{i}(i=1, \ldots, r)$ are the first $r$ components of $F$.

When $r=1$ or $2, \bar{A}(\mu)$ at $\mu=0$ is

$$
\text { [0], }\left[\begin{array}{ll}
0 & 0 \\
0 & 0
\end{array}\right] \text { or }\left[\begin{array}{ll}
0 & 1 \\
0 & 0
\end{array}\right] \text {; }
$$

then we find that the equation (5.3) for small solutions will be just the equations we discussed in Sections 2, 3 and 4 respectively, and the results established there are valid. Therefore if $A$ has a simple eigenvalue at $\mu=0$, no bifurcating nonconstant periodic solution can exist, while for a double eigenvalue a nonconstant periodic solution with period $O(1 / \mu)$ for $\mu \rightarrow 0$ may exist.

If $r>2$, suitable theorems can be proved [6] to show that bifurcating nonconstant periodic solutions with period $O(1 / \mu)$ for $\mu \rightarrow 0$ exist.

Now we demonstrate by example that in several cases both a periodic and steady bifurcating solution may exist together (see the discussion in Section 3).

Examples. In (5.3) let

$$
y=\varepsilon(u, v, w)
$$

then, in the same way as in Section 3, we find that if $\bar{F}(y)$ begins with a cubic nonlinearity, we must set

$$
s=\varepsilon^{2} t, \quad \mu=\varepsilon^{2} v .
$$

1. Consider

$$
\left.\begin{array}{l}
\frac{d u}{d s}=v \alpha u-(u+v)\left(u^{2}+v^{2}\right), \\
\frac{d v}{d s}=v \alpha v+(u-v)\left(u^{2}+v^{2}\right), \quad \alpha, \beta>0, \\
\frac{d w}{d s}=v \beta w-w\left(u^{2}+v^{2}+w^{2}\right) .
\end{array}\right\}
$$

This time $A$ has a triple eigenvalue at $\mu=0$ and the null solution loses its stability as the real eigenvalues $\mu \alpha$ and $\mu \beta$ pass from negative to positive values. Notice that the first two equations are the same as Example 1 in Section 3.

This system has a nontrivial steady solution curve, namely $u=v=0$ and $w= \pm \sqrt{ }(v \beta)$ for $v>0$, which is unstable. Therefore (5.1) also has a unique supercritical unstable steady solution in the neighbourhood of $(0,0, \pm \sqrt{ }(\mu \beta), 0, \ldots, 0)$. 
As well as this solution, equation (5.4) has a periodic solution $\bar{u}^{2}+\bar{v}^{2}=v \alpha, \bar{w}=0$ with period $2 \pi / v \alpha$ in $s$. The stability of this solution will be determined by the variational system

$$
\left[\begin{array}{l}
\frac{d \phi}{d s} \\
\frac{d \psi}{d s} \\
\frac{d \xi}{d s}
\end{array}\right]=\left[\begin{array}{ccc}
\left(v \alpha-3 \bar{u}^{2}-\bar{v}^{2}-2 \bar{u} \bar{v}\right) & \left(-3 \bar{v}^{2}-\bar{u}^{2}-2 \bar{u} \bar{v}\right) & 0 \\
\left(3 \bar{u}^{2}+\bar{v}^{2}-2 \bar{u} \bar{v}\right) & \left(v \alpha-3 \bar{v}^{2}-\bar{u}^{2}+2 \bar{u} \bar{v}\right) & 0 \\
0 & 0 & v \beta
\end{array}\right]\left[\begin{array}{l}
\phi \\
\psi \\
\zeta
\end{array}\right]
$$

One Floquet multiplier is one; another is $e^{-4 \pi}<1$ (just that given in Example 1 of Section 3) and the third is $e^{2 \pi \beta / \alpha}>1$ for $\beta / \alpha>0$. Therefore the full equation (5.1) has a supercritical periodic solution in the neighbourhood of

$$
(\sqrt{ }(\mu \alpha) \cos (\mu \alpha t), \sqrt{ }(\mu \alpha) \sin (\mu \alpha t), 0,0, \ldots, 0), \quad \mu>0,
$$

with period close to $2 \pi / \mu \alpha$, and this solution is orbitally unstable.

2. Consider

$$
\begin{aligned}
& \frac{d u}{d s}=v \alpha u+v \gamma v-u\left(u^{2}+v^{2}\right), \\
& \frac{d v}{d s}=-v \gamma u+v \alpha v-v\left(u^{2}+v^{2}\right), \quad \alpha, \beta>0, \\
& \frac{d w}{d s}=v \beta w-w\left(u^{2}+v^{2}+w^{2}\right),
\end{aligned}
$$

Again $A$ has a triple eigenvalue at $\mu=0$ and the null solution loses its stability as a complex conjugate pair $\mu(\alpha \pm i \gamma)$ and the real $\mu \beta$ pass from the left to the right half of the complex plane. Note that the first two equations are the same as Example 2 in Section 3.

The system (5.1) will then have a unique supercritical steady bifurcating solution in the neighbourhood of $(0,0, \pm \sqrt{ }(\mu \beta), 0, \ldots, 0)$, which is unstable. There also exists a bifurcating supercritical periodic solution in the neighbourhood of

$$
(\sqrt{ }(\mu \alpha) \cos (\mu \gamma t),-\sqrt{ }(\mu \alpha) \sin (\mu \gamma t), 0,0, \ldots, 0), \quad \mu>0,
$$

with period close to $2 \pi / \mu \gamma$. This solution is orbitally unstable.

For $r \geqslant 3$, the general categorization of the number of periodic solutions can no longer be calculated because Bendixon's theorem and phase plane techniques are no longer available. For further discussion see [6].

The techniques outlined here also apply to a partial differential equation in a Hilbert space, if $A$ has compact resolvent. Then using semigroup theory of operators, 
all but $r$ of the equations may be eliminated so that a system of $r$ ordinary differential equations, like (5.3), need to be studied (see [6]).

Finally, we remark that the results due to Freedman [3] that no bifurcating periodic solutions exist with period $T(\mu) \rightarrow T_{0} \neq 0$ as $\mu \rightarrow 0$ can be extended to a system of equations in $\mathbf{R}^{n}$ or to a Hilbert space. However, Freedman's proof depends on the fact that each limit cycle in the plane has a critical point in its interior, and therefore his methods cannot be appiied to the case of $A$ having an eigenvalue of multiplicity $r \geqslant 2$ at $\mu=0$. A proof which does take this into account is given in [6].

\section{References}

[1] E. A. Coddington and N. Levinson, Theory of ordinary differential equations (McGraw-Hill, New York, 1955).

[2] W. A. Coppel, “A survey of quadratic systems", J. Differential Equations 2 (1966), 293-306.

[3] H. I. Freedman, "The nonbifurcation of periodic solutions when the variational matrix has a zero eigenvalue", J. Math. Anal. Applic. 51 (1975), 429-439.

[4] J. Hale, Ordinary differential equations (Wiley-Interscience, New York, 1969).

[5] E. Hopf, "Abzweigung eines periodischer Losung eines Differential systems", Bericheten der Mathematisch Physikalischen Klasse der Sächsischen Akademie der Wissenschaften, Leipzig XCIV (1942), 1-22.

[6] K. A. Landman, Bifurcation and stability of solutions (University of Melbourne: Ph.D. thesis, 1978).

[7] K. A. Landman and S. Rosenblat, "Bifurcation from a multiple eigenvalue and stability of solutions", SIAM Applied Math, 34 (1978), 743-759.

[8] G. Sansone and R. Conti, Nonlinear differential equations (Pergamon Press, Oxford, 1964).

[9] M. Urabe, Nonlinear autonomous oscillations (Academic Press, New York, 1967).

\section{Department of Mathematics}

University of Melbourne

Parkville, Vic., 3052

Australia 